\title{
Thermoluminescence properties of nanostructured calcium borate as a sensitive radiation dosimeter for high radiation doses
}

\begin{abstract}
The crystalline calcium tetraborate ( $\mathrm{CaB} 4 \mathrm{O} 7)$ nanoparticles were synthesized using a combination of facile co-precipitation and thermal treatment. The synthesized phosphor nanoparticles were found to possess a monoclinic nanostructure of particle size of about 8 $\mathrm{nm}$. The thermoluminescence (TL) glow curve of the nanoparticles shows a single peak centred at about $150^{\circ} \mathrm{C}$. The TL nanophosphor revealed an excellent dosimetric response with a respectable linearity in the dose range of 0.05 to $1000 \mathrm{~Gy}$, which is wider than its counterparts prepared by non nanosynthesis methods. They exhibited good luminescence efficiency and wide range linearity, suggesting the present phosphor nanoparticles may be considered as a suitable candidate for the dosimetric applications.
\end{abstract}

Keyword: Calcium borate; Nanocrystal; Thermoluminescence; Co-precipitation; Radiation dose 